\title{
Instrumentos para la gestión de tierra vacante en ciudades
}

\section{argentinas $\left[{ }^{[1]}\right.$}

\section{Instruments for vacant land management in Argentinian cities.}

Instrumentos para
gerenciamento de terras
vagas em cidades argentinas

Instruments pour la gestion des terres vacantes dans les villes argentinees

\section{Daniela Cortizo}

Instituto de Investigaciones y Políticas del Ambiente Construido, Facultad de Arquitectura y Urbanismo, Universidad Nacional de La Plata, Consejo Nacional de Investigaciones Científicas y Técnicas (IIPAC-FAU-UNLP-CONICET)

cortizodaniela@gmail.com https://orcid.org/0000-0001-8953-3978

\section{Cómo citar este artículo:}

Cortizo, D., Rodríguez Tarducci, R. y Frediani, J. (2021). Instrumentos para la gestión de tierra vacante en ciudades argentinas. Bitácora Urbano Territorial, 31(III): 109-122. https://doi.org/ 10.15446/bitacora.v31n3.87415

[1] El artículo tiene su origen en la tesis "Tierra vacante: Estado y mercado en los procesos de crecimiento urbano. Estrategias para su gestión en el Partido de La Plata". Doctorado en Geografía, FaHCE, UNLP, Argentina.

Instituto de Investigaciones y Políticas del Ambiente Construido, Facultad de Arquitectura y Urbanismo, Universidad Nacional de La Plata, Consejo Nacional de Investigaciones Científicas y Técnicas (IIPAC-FAU-UNLP-CONICET) http://orcid.org/0000-0002-7238-1979 


\section{Resumen}

Las ciudades argentinas se caracterizaron en las últimas décadas por un aumento acelerado de la superficie urbanizada, en el marco de una creciente necesidad de suelo. Estos procesos de crecimiento urbano dan lugar a ciudades que crecen y se expanden sobre tierras vacantes, y a su vez las generan en su interior, extendiendo indefinidamente los límites urbanos, lo que trae aparejado una gran cantidad de problemas. En este contexto, se considera que la existencia de tierras vacantes al interior de estas ciudades es resultado de la combinación entre funcionamiento especulativo del mercado, con lógicas no reguladas, y las restricciones en la gestión del crecimiento urbano por parte del Estado. Es por esto que se propone reconstruir la caja de herramientas disponibles con la que cuenta el Estado para actuar sobre el fenómeno de la vacancia del suelo, en pos de (re)orientar los actuales procesos de crecimiento urbano. Para tal fin, se relevan y analizan los distintos mecanismos, instrumentos, prácticas y procedimientos con los que cuenta el Estado - en sus distintos nivelespara accionar sobre la problemática.

Palabras Clave: gestión, ciudad, urbanización

\section{Autoras}

\section{Daniela Cortizo}

Doctora en Geografía (año 2020), Magister en Ciencias del Territorio (año 2018) y Arquitecta (año 2014) de la Universidad Nacional de La Plata (UNLP), Buenos Aires, Argentina. Actualmente Becaria Posdoctoral en CONICET en el Instituto de Investigaciones y Políticas del Ambiente Construido (IIPAC-CONICET-UNLP). Ha participado, expuesto y organizado congresos nacionales e internacionales, publicado artículos en revistas científicas, ejercido la docencia en cátedras de planeamiento urbano y colaborado en el desarrollo de planes de ordenamiento territorial en distintas ciudades argentinas.

\section{Julieta Frediani}

Doctora en Geografía de la Universidad Nacional de La Plata (UNLP). Licenciada y Profesora en Geografía. Especialista en Ciencias del Territorio de la Facultad de Arquitectura, UNLP. Investigadora Adjunta del CONICET. Profesor en el Grado y Posgrado en la UNLP.

\section{Rocío Rodríguez Tarducci}

Doctora en Geografía (año 2020), Arquitecta (año 2014) de la Universidad Nacional de La Plata (UNLP), Buenos Aires, Argentina. Actualmente Becaria Posdoctoral en CONICET en el Instituto de Investigaciones y Políticas del Ambiente Construido (IIPAC-CONICET-UNLP) y se desempeña como docente en la Universidad Nacional Arturo Jauretche (UNAJ). Ha participado, expuesto y organizado congresos nacionales e internacionales, publicado artículos en revistas científicas y ejercido la docencia en cátedras de planeamiento urbano. 


\section{Abstract}

In recent decades, Argentine cities have been characterized by an accelerated increase in urbanized areas, in the context of a growing need for land. These urban growth processes give rise to cities that grow and expand on vacant land, and at the same time generate them inside, indefinitely extending urban limits, which brings with it a large number of problems. In this framework, it is considered that the existence of vacant land within these cities is the result of the combination between speculative market operation, with unregulated logics, and restrictions on the management of urban growth by the State. For this reason, it is proposed to rebuild the toolbox available to the State to act on the phenomenon of land vacancy, in order to (re) guide the current processes of urban growth. To that end, the different mechanisms, instruments, practices and procedures available to the State -at its different levels - to act on the problem are surveyed and analyzed.

Keywords: : management, cities, urbanization

\section{Résumé}

Les villes argentines ont été caractérisées au cours des dernières décennies par une augmentation accélérée de la zone urbanisée, dans le contexte d'un besoin croissant de terres. Ces processus de croissance urbaine donnent naissance à des villes qui se développent sur des terrains vacants, et à leur tour les génèrent à l'intérieur, prolongeant indéfiniment les limites urbaines, ce qui entraîne un grand nombre de problèmes. Dans ce cadre, on considère que l'existence de terrains vacants dans les villes argentines est le résultat de la combinaison entre un fonctionnement spéculatif du marché, avec des logiques non réglementées, et des restrictions à la gestion de la croissance urbaine par l'État. Pour cette raison, il est proposé de reconstruire la boîte à outils disponible à la disposition de l'Etat pour agir sur le phénomène de la vacance foncière, afin de (re) guider les processus actuels de croissance urbaine. À cette fin, les différents mécanismes, instruments, pratiques et procédures dont l'État dispose - à ses différents niveaux - pour agir sur le problème sont recensés et analysés.

\section{Resumo}

As cidades argentinas foram caracterizadas nas últimas décadas por um aumento acelerado da área urbanizada, no contexto de uma crescente necessidade de terras. Esses processos de crescimento urbano dão origem a cidades que crescem e se expandem em terrenos vagos e, por sua vez, o geram, estendendo indefinidamente os limites urbanos, o que traz consigo um grande número de problemas. Nesse contexto, considera-se que a existência de terrenos baldios nas cidades argentinas é o resultado da combinação entre operações especulativas de mercado, com lógicas não regulamentadas e restrições à gestão do crescimento urbano pelo Estado. Por esse motivo, propõe-se reconstruir a caixa de ferramentas disponível ao Estado para atuar no fenômeno da vacância fundiária, a fim de (re) orientar os processos atuais de crescimento urbano. Para tanto, são levantados e analisados os diferentes mecanismos, instrumentos, práticas e procedimentos que o Estado possui - em seus diferentes níveis - para atuar no problema.

\section{Palavras-chave:gerenciamento, cidade, urbanização}

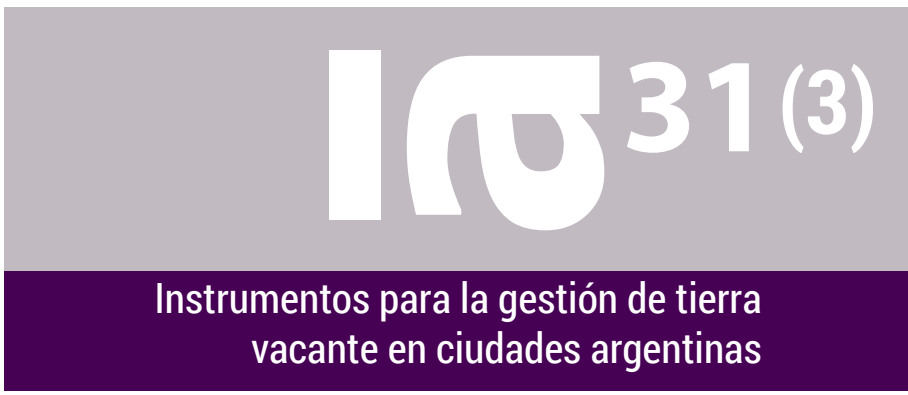

Mots-clés: gestion, ville, urbanisation 


\section{Introducción}

Las ciudades argentinas se han caracterizado en las últimas décadas por un aumento acelerado de la superficie urbanizada, en el marco de una creciente necesidad de suelo, donde el capital privado es protagonista del desarrollo urbano. Estos procesos de crecimiento urbano dan lugar a ciudades que crecen y se expanden sobre tierras vacantes, y a su vez las generan en su interior, extendiendo indefinidamente los límites urbanos, lo que trae consigo una gran cantidad de problemas. En el presente trabajo se entiende por tierra vacante aquella tierra que permanece no utilizada o subutilizada, con límites precisos, que puede encontrarse desocupada o con alguna edificación, y que se encuentra localizada dentro de los límites urbanos o del área urbanizable ${ }^{[2]}$, pudiendo ser de propiedad privada o fiscal.

los municipios del país

cuentan con potestades

para regular el mercado

de suelo. Sin embargo,

por debilidades insti-

tucionales, carencia de

conocimientos técnicos

o falta de instrumentos

vigentes, la mayoría

de ellos no las utiliza o

lo hace parcialmente"

(Lanfranchi et al., 2018,

p. 242).
Las ciudades crecen extendiéndose sobre tierras vacantes cada vez más alejadas, sin buena dotación de infraestructura, servicios y equipamientos, mientras al interior del área urbana existen otras tierras vacantes que sí poseen estas características. Es por esto que se considera que su existencia implica un gran desaprovechamiento desde el punto de vista económico, social y ambiental, ya que trae aparejados grandes costos para el Estado en lo que respecta a la provisión de infraestructura, equipamiento, transporte y servicios para estas nuevas urbanizaciones. De este modo, se extienden los límites de las ciudades, tanto en los aspectos formales (la ampliación de las áreas urbanizables en los códigos de ordenamiento urbano), como en los casos concretos de generación de nuevos barrios por fuera de estos límites legales.

En este marco, se considera que la existencia de tierras vacantes es resultado de la combinación entre el funcionamiento especulativo del mercado, con lógicas no reguladas, y las restricciones en la gestión del crecimiento urbano por parte del Estado. Estas restricciones se encuentran en la desarticulación de distintos niveles del Estado - como también de organismos y políticas al interior de estos-, en la limitación de recursos - tanto económicos como humanos-, en las debilidades en la utilización de instrumentos para la regulación del mercado de suelos y en la complejidad propia de la planificación y gestión urbana - que se realiza en ámbitos de incertidumbre en donde la ciudad es una sola, aunque en ella intervengan distintas jurisdicciones y normativas y existan una diversidad de actores que pujan por sus intereses.

Es por esto que se considera fundamental la gestión estatal de estos espacios vacantes, en pos de (re)utilizarlos. De ese modo, el objetivo del presente artículo es analizar los distintos instrumentos disponibles con los que cuenta el Estado para accionar sobre la problemática de la tierra vacante en las ciudades argentinas. Para cumplir con el objetivo se propone reconstruir la caja de herramientas disponibles, a partir del relevamiento y análisis de los mecanismos, prácticas y procedimientos con los

[2] El área urbanizable es el área de la ciudad en donde la normativa de usos de suelo vigente permite usos urbanos. 
que cuenta el Estado - en sus distintos niveles - para accionar sobre el fenómeno de la vacancia del suelo, en pos de (re)orientar los actuales procesos de crecimiento urbano.

En cuanto a la estructura del artículo, el primer apartado analiza el contexto latinoamericano y argentino con relación a los procesos de crecimiento urbano. En el segundo apartado se focaliza la problemática de la tierra vacante en las ciudades y su relación con el accionar del Estado y el mercado, poniendo énfasis en la gestión del suelo en Argentina. En el tercer apartado se analizan los distintos instrumentos con los que cuenta el Estado para accionar sobre la problemática de estudio. Por último, se hacen algunas reflexiones finales.

\section{Ciudades Argentinas Com-Fusas y Periferias Vacantes}

Los países latinoamericanos han experimentado en las últimas décadas transformaciones derivadas de los procesos de globalización y reestructuración económica, iniciados durante el último cuarto del siglo $X X$, que modifican su estructura espacial y social (De Mattos, 2002; Borja y Castells, 2000). Los efectos urbanos de estos procesos, es decir, los efectos de lo global en lo local, se relacionan con el crecimiento de las ciudades ${ }^{[3]}$.

Estas ciudades, que crecen cada vez más, lo hacen con rasgos comunes como la concentración en grandes urbes, la existencia de grandes áreas con déficit en la cobertura de servicios básicos y de grandes desigualdades en el acceso al suelo y a la vivienda $-y$, por consiguiente, a los principales recursos urbanos - , y la segregación socio espacial (Luco Simioni, 2001). De este modo, América Latina se configura como una de las regiones más urbanizadas del mundo, en donde el $81 \%$ de la población vive en ciudades (Banco Mundial, 2018).

En este marco, la República Argentina es uno de los países más urbanizados de la región: en las últimas décadas, la población urbana ascendió del $74 \%$, en 1960, al 92\% en 2017 (Banco Mundial, 2018), generando un fenómeno de transformación de las ciudades, a

[3] Se considera a la ciudad como el escenario de conflicto donde existe una multiplicidad de actores con distintos intereses que compiten por la tierra urbana, recurso esencial para la existencia de las ciudades. través de nuevas formas de crecimiento urbano. Estos procesos tienen dos características significativas, que se encuentran relacionadas con el aumento de la superficie urbanizada y del consumo de suelo urbano por habitante (Lanfranchi et al., 2018).

En el período comprendido entre 1991 y 2010, gran cantidad de ciudades han incrementado su superficie en porcentajes mucho más elevados que su población; es el caso de Mendoza (-5\% crecimiento poblacional, $34 \%$ crecimiento de la superficie urbanizada), Córdoba ( $8 \%$ crecimiento poblacional, $51 \%$ crecimiento de la superficie urbanizada), Rosario (4\% crecimiento poblacional, $33 \%$ crecimiento de la superficie urbanizada), La Plata (21\% crecimiento poblacional, $127 \%$ crecimiento de la superficie urbanizada), Mar del Plata $(16 \%$ crecimiento poblacional, $115 \%$ crecimiento de la superficie urbanizada), entre otras (Uccellatore et al., 2015). Este crecimiento se produjo extendiendo la superficie de las ciudades de forma dispersa y con baja densidad en las periferias, con presencia de gran cantidad de espacios no utilizados o subutilizados al interior: las tierras vacantes.

El urbanismo posmoderno neoliberal es el paradigma dominante en el manejo de estas ciudades, en donde se agudizan las tendencias de mercantilización y el capital privado es protagonista del desarrollo urbano (De Mattos, 2002). De este modo, las periferias de las ciudades son cada vez más extensas y se encuentran bajo la especulación y presión inmobiliaria, sujetas a la apropiación privada de plusvalías, sobre todo en relación con la presencia de estas tierras sin uso.

En este sentido, es importante reconocer el papel de la tierra como uno de los principales recursos necesarios para la existencia de las ciudades y el desarrollo de la vida urbana (Baer, 2011). En la ciudad neoliberal, además, posee un rol preponderante en la formación de riqueza de los sectores dominantes y en la formación de una cultura patrimonialista de la sociedad (Reese, 2018). De este modo, la tierra urbana se convierte en el espacio de disputa entre los actores que intervienen en los procesos de crecimiento urbano.

Una de las consecuencias de la predominancia del mercado, en materia de desarrollo urbano, es que se producen ciudades que conjugan de forma simultánea procesos de densificación y expansión, que se compactan y se difunden: ciudades com-fusas (Abramo, 2012). Estas se conforman, por un lado, por la ciudad compacta, a partir de procesos de densificación 
que se dan mayoritariamente en los centros urbanos; y, por el otro, por la ciudad difusa, a partir de procesos de expansión que se dan en las periferias.

Esta ciudad es producida tanto por el mercado informal (que la compacta con la densificación de villas y la difunde con la expansión de asentamientos informales), como por el formal (que la compacta a partir de la densificación con torres y la expande con la creación de nuevos loteamientos y urbanizaciones cerradas). Este mercado dirige sus inversiones hacia donde tiene demanda solvente, movilizado por una lógica de maximización de la ganancia. En este marco, estas ciudades se caracterizan por altísimos precios del suelo urbanizado, apropiación concentrada de las rentas, elevados costos para la provisión de infraestructura y servicios y escasez de oferta formal de suelo urbano de calidad (Reese, 2018).

En muchos casos, la expansión de estas ciudades se da de manera desfasada con relación a la dotación de servicios, infraestructura y equipamientos (Uccellatore et al., 2015) producto del crecimiento acelerado que han tenido las periferias. A su vez, en muchos casos se extienden sobre áreas con riesgo hídrico, es decir, áreas productivas (compitiendo por el suelo) por fuera de los límites establecidos por las normativas urbanísticas, con gran cantidad de familias que habitan en asentamientos informales y gran cantidad de urbanizaciones cerradas, lo que pone en evidencia un proceso de crecimiento marcado por las desigualdades.

Este proceso de crecimiento está caracterizado por una gran cantidad y diversidad de tierras vacantes. Estos espacios constituyen un porcentaje importante de las áreas urbanas latinoamericanas en general, y argentinas en particular, en donde representan en promedio el 23.4\% de su superficie (Uccellatore et al., 2015) ${ }^{[4]}$. De este modo, se configuran las 'periferias vacantes' no solo de usos, sino también de infraestructura, servicios, equipamientos y centralidad y, en muchos casos, degradadas ambientalmente (Cortizo, 2018).

\section{Tierra Vacante: de Problema a Oportunidad}

Las tierras vacantes se localizan tanto en la ciudad difusa como en la compacta, y la existencia de estas tierras ha definido las formas que ha adoptado el cre-

[4] En las ciudades de Argentina con más de 20.000 habitantes, un promedio del $23.4 \%$ de la superficie de las zonas urbanas parceladas son baldíos.

114 | Bitācora 31(3) | septiembre-diciembre 2021 cimiento de las ciudades (Clichevsky, 2007), ya que muchas han incorporado tierra 'produciendo' loteos salteados, dejando tierra intersticial sin uso y, en muchos casos, ocupando otras ambientalmente degradadas. En función de esto cabe preguntarse, por un lado, por qué se generan estas tierras, es decir, qué condiciones permiten su existencia y permanencia; $y$, por el otro, quiénes la producen, ocupan y gestionan, es decir, quiénes son los actores involucrados.

Para dar respuesta a estos dos interrogantes, en primer lugar, es importante considerar las diversas condiciones que pueden darle origen a la vacancia. Se considera una problemática multicausal, ya que existe una diversidad de causas que dan origen al fenómeno (Frediani, 2014). Puede tratarse de condiciones legales, en relación con la estructura de propiedad y tenencia de la tierra, herencias vacantes, tierras abandonadas; económicas, en relación con la retención especulativa o patrimonial como modo de ahorro familiar o falta de recursos económicos para movilizarlas; espaciales-funcionales (tamaño, localización o falta de servicios); ambientales (localización en áreas inundables), o de problemas propios de gestión (edificios públicos abandonados, que pueden ser militares, portuarios, entre otros).

En segundo lugar, la tierra urbana, y en particular la que se encuentra vacante, es espacio de disputa, por lo que existe una gran diversidad de actores que intervienen en los procesos de crecimiento que tienen distintas lógicas e intereses; en ese sentido, se genera tierra vacante por el Estado tanto como por el mercado. El mercado la genera porque entiende la tierra como una mercancía. Esto es posible porque tiene características propias que la diferencian de otros bienes transables en el mercado (Barenboim, 2013; Baer, 2011). Primero, es una mercancía única e irreproducible, por la incapacidad de que haya dos tierras iguales, con igual localización. Segundo, es escasa, de forma física (presencia insuficiente de tierras urbanizadas), económica (disponibilidad efectiva de tierras ofertadas) o jurídica (por restricciones en la regulación). Tercero, es imperecedera, es decir que no se destruye, tiene duración a largo plazo. Esta cualidad brinda la posibilidad de acumular riqueza.

Estas características, y el hecho de que el precio de la tierra se defina por factores externos a ella (ubicación en relación a la ciudad, normativa vigente, servicios disponibles), hacen que el mercado genere tierra vacante a partir de la especulación, que es la actitud o estrategia que consiste en mantener tierra vacante a la 
espera de que su valor aumente a causa de la acción del Estado u otros factores. De este modo, se retiene la tierra, lo que produce menor oferta en el mercado, y se incrementan los precios.

Pero el Estado también propicia condiciones para la generación de tierra vacante, tanto por su acción como por su inacción. Por un lado, la acción del Estado incrementa los precios del suelo, a partir de la modificación de normas urbanísticas (aumento de indicadores, ampliación de áreas urbanas, modificación de usos y formas de subdivisión del suelo), la extensión de infraestructuras, la creación de equipamientos o el anuncio de políticas urbanas. En muchos casos esto promueve la retención de tierras, produciendo ganancias extraordinarias para sus propietarios, sobre todo cuando desde el Estado no se capta la valorización. Por otro lado, la inacción del Estado también genera tierra vacante debido a la ausencia de políticas integrales, la no utilización de instrumentos de captación de plusvalías, y la no utilización de instrumentos de gestión del suelo que permitan movilizarlas. De este modo, la existencia de tierras vacantes pone en evidencia la acentuada debilidad e intervención del Estado y un predominio del mercado en la producción de ciudad. Así, la tierra vacante se convierte en un problema, porque representa desaprovechamiento en términos económicos, sociales, ambientales y funcionales.

En términos económicos, la ciudad se extiende cada vez más y va generando nuevas tierras vacantes, lo que implica grandes costos para el Estado como proveedor de infraestructura, servicios y equipamientos; a su vez, existen tierras desaprovechadas que poseen buena dotación de servicios e infraestructura. En términos sociales, las tierras vacantes implican este desaprovechamiento, ya que evidencian la existencia de tierra apta para utilizar en contraposición a la demanda habitacional. Y, en términos ambientales y funcionales, implican discontinuidades en la trama urbana y problemas ambientales por falta de mantenimiento.

En este sentido, es el Estado el que puede transformar este problema en una oportunidad, entendiendo estos espacios como lugares estratégicos con gran potencial para redireccionar los actuales procesos de crecimiento urbano que conforman la ciudad com-fusa. De igual manera, la tierra sería comprendida como un recurso que puede ser movilizado para atender problemáticas habitacionales, funcionales y ambientales, siendo receptora de la implementación de políticas a partir de la utilización de instrumentos de gestión. La gestión de la tierra vacante es un aspecto esencial de la gestión del crecimiento urbano, que considera que, sin Estado, la tierra vacante es para el mercado.

\section{La Gestión del Suelo en Argentina}

Los procesos de crecimiento que se dan en las ciudades responden a decisiones de actores de diferentes escalas: locales, provinciales, nacionales y globales. En Argentina, son los gobiernos locales quienes ejercen un mayor control en la regulación y planificación del crecimiento urbano. La regulación del uso del suelo presenta una gran heterogeneidad espacial a nivel local, con variaciones sustanciales entre ciudades (Goytia, Pasquini, 2012, en Goytia, Cristini, 2017).

En este contexto, a partir de la pregunta por cómo puede abordarse el problema de la vacancia en las ciudades argentinas, surge, en primer lugar, la necesidad de considerar las distintas jurisdicciones que operan en una ciudad en donde el Estado tiene distintos actores con competencia en la regulación y producción del suelo en sus distintos niveles. También hay que considerar la existencia de distintos marcos regulatorios y políticas que impactan en la configuración de estas ciudades.

A nivel nacional existen normativas y políticas que abordan diversas cuestiones relacionadas con la configuración urbana y la generación de tierras vacantes. En este nivel se establecen los marcos regulatorios generales, el régimen de propiedad y dominio, y se impulsan políticas de vivienda, que tienen distintos niveles de impacto en las ciudades. Al no contar en la actualidad con una ley integral a nivel nacional, la regulación y ordenamiento de usos del suelo se rige en el país por legislaciones y regulaciones sectoriales, dispersas y desarticuladas ${ }^{[5]}$.

A nivel provincial se establecen las normativas en relación con los aspectos ambientales y tributarios, y solo en pocas provincias (Buenos Aires, Mendoza) se regula en este nivel el ordenamiento general de uso del suelo (a excepción de la Ciudad Autónoma de Buenos Aires que lo hace a nivel local).

\footnotetext{
[5] En el año 2012 se impulsó desde el gobierno nacional un Anteproyecto de Ley de Planificación y Ordenamiento Territorial, como norma marco para todo el país, pero no prosperó.
} 
A nivel municipal se establecen normativas urbanas (de forma obligatoria en las ciudades que se localizan en estas provincias que poseen leyes de ordenamiento urbano, y opcional en las demás ciudades) y planes estratégicos. Es decir que la escala local es la que tiene mayor incidencia en la regulación del crecimiento urbano. A partir del análisis de los marcos regulatorios de los distintos niveles del Estado en relación con la temática de estudio, se reconocieron distintos instrumentos para accionar sobre la vacancia del suelo.

\section{Instrumentos para la Gestión de Tierra Vacante: la Caja de Herramientas del Estado}

La gestión de la tierra vacante es la manera de ocuparse del no uso del suelo en una ciudad; los instrumentos para su gestión son las herramientas, mecanismos y procedimientos que dispone el municipio para intervenir en sus territorios y que, en conjunto, se constituyen como la caja de herramientas del Estado para accionar sobre el fenómeno de la vacancia del suelo.

De este modo, en función de las políticas urbanas, se diseñan y ponen en práctica diferentes instrumentos de regulación para que la ciudad no sea el resultado estricto de las lógicas del mercado (Barenboim, 2013). Resulta importante entender que los instrumentos a utilizar tienen que estar en relación con los objetivos que se quieren conseguir. Por eso, es necesario contar con un diagnóstico adecuado que reconozca las tierras vacantes existentes y las condiciones que les dieron origen, para saber cuáles son los instrumentos más adecuados en cada caso, pues su uso articulado y combinado garantiza su eficacia.

Se analizan a continuación distintos instrumentos, desde una mirada transformadora y activa del Estado, orientados al fortalecimiento de su rol como regulador del suelo. Los instrumentos se pueden clasificar en instrumentos para movilizar, para adquirir tierra para el municipio, para administrar tierra del municipio y, por último, para planificar ${ }^{[6]}$ (Tabla 1).

[6] Cabe aclarar que muchos de los instrumentos analizados se pueden utilizar para gestionar procesos que van más allá de la tierra vacante o el crecimiento urbano, pero para efectos de esta investigación se los vinculará con la problemática estudiada.

\section{Instrumentos para movilizar Tierra Vacante}

Estos instrumentos son aquellos que puede usar el municipio para tener un rol activo en la utilización de estos espacios; puede tratarse de instrumentos para facilitar, incentivar o exigir la movilización. En primer lugar, los instrumentos para exigir la movilización se ejecutan a partir de la imposición de construir, subdividir o urbanizar en un determinado plazo de tiempo. Entre ellos se pueden mencionar los siguientes:

- Edificación y/o parcelamiento obligatorio: permite exigirle al propietario de una tierra vacante la obligación de parcelarla y/o edificarla en un plazo determinado. Puede ser utilizado para que los municipios tengan un rol activo en la utilización de tierras que estén localizadas en áreas consolidadas con buena dotación de infraestructura. Su objetivo es promover el uso eficiente de la misma, "que el Estado contribuyó a desarrollar mediante la inversión de recursos públicos, al ampliar la disponibilidad de suelo urbano (...) En efecto, al optimizar la infraestructura básica instalada, contribuye a una planificación y un ordenamiento territorial más eficiente" (Escales et al., 2017, p. 33). En caso de incumplimiento, este instrumento puede combinarse con la aplicación de gravámenes de carácter progresivo y, en caso de no cumplimentarse, se puede proceder a declarar la tierra de utilidad pública y expropiar.

- Declaración de zona de desarrollo prioritario: puede implementarse en determinadas áreas de la ciudad y tiene como objetivo revertir la situación de vacancia, fomentar el desarrollo de edificaciones paralizadas, impulsar la reorganización parcelaria y/o promover la concreción de determinados usos, permitiendo a los gobiernos locales establecer plazos para urbanizar y/o edificar. Para lograr una mayor eficacia, este instrumento puede combinarse con la aplicación de alguna herramienta fiscal que grave específicamente la ociosidad.

En segundo lugar, los instrumentos para facilitar la movilización tienen como objetivo simplificar la ejecución de proyectos sobre estos espacios, promoviendo el uso eficiente de la infraestructura. Entre estos se pueden mencionar los siguientes:

- Reajuste de tierras: se utiliza para reconfigurar espacios urbanos, optimizarlos y darles mejor funcionalidad. Tiene por objetivo posibilitar la ejecución de emprendimientos urbanísticos en áreas formadas por dos o más tierras vacantes de dife- 
rentes propietarios, por medio de la modificación de su estructura parcelaria y jurídica (Escales et al., 2017). Al mismo tiempo, es un instrumento de carácter económico, ya que supone fortalecer los procesos de financiamiento del desarrollo urbano y acordar en cada intervención esquemas de distribución de cargas y beneficios.

- Consorcios o convenios urbanísticos: permiten, al propietario de la tierra vacante que no esté en condiciones financieras de emprender obras, asociarse con el Estado y otros actores privados para ejecutar proyectos de urbanización o edificación. Resulta interesante que se encuentren articulados con la recuperación de plusvalías, pues tienen objetivos de tipo redistributivos.

- Lote con servicios: facilita la generación y acceso a las tierras vacantes, en particular a las que se localizan en áreas en crecimiento de las ciudades, permitiendo que los servicios lleguen progresivamente, por lo que en el precio inicial de la tierra no está esa carga.

- Concesión de uso: es el contrato administrativo mediante el cual quien ejerce el dominio de la tierra vacante (el Estado o un privado) acuerda el uso de la misma durante un período determinado, recibiendo un canon $\mathrm{u}$ otra contraprestación que se fija previamente.

En tercer lugar, los instrumentos para incentivar la tenencia de tierras vacantes tienen como objetivo desalentar la especulación inmobiliaria, para que los propietarios construyan $u$ ofrezcan el bien en el mercado.

- Impuesto: se puede aplicar como disuasivo de la especulación (Lanfranchi et al., 2018); para ello debe ser lo suficientemente elevado, con el objetivo de incentivar al propietario a utilizar la tierra u ofrecerla en el mercado (Perelman et al., 2017). Puede aplicarse como un impuesto progresivo en el tiempo, "como propuesta de desestimulo a los comportamientos especulativos y de captura, por parte de los propietarios, de las plusvalías generadas a partir de inversiones públicas, en su forma tradicional" (Larangeira, 2004, p. 15). De este modo, aumentando el costo de posesión del bien, se intenta trasmitir al propietario que le resultará más costoso mantenerlo en desuso que utilizarlo o venderlo. Deberían gravarse con mayor carga aquellas tierras que posean buena dotación de infraestructura y equipamiento (áreas centrales), a diferencia de aquellos que presentan carencias de estos (áreas periféricas) (Uccellatore et al., 2015). Pueden ser tributos al suelo libre de mejoras, sobre-alícuotas al suelo baldío, alícuotas progresivas, multas, entre otros, y, para su eficacia, necesitan de la articulación entre las políticas de suelo y las tributarias (entre los organismos de planificación urbana y los de recaudación).

En este punto es importante señalar que aplicar instrumentos de castigo, como el aumento de cargas impositivas para disuadir la retención de tierras, no necesariamente resuelve el problema de la especulación. Por eso es importante combinarlo con otros instrumentos que, por ejemplo, permitan al propietario elegir entre un castigo o un beneficio, en caso de revertir la situación de vacancia, premiando la movilización a partir de incentivos económicos o fiscales.

\section{Instrumentos para adquirir Tierra Vacante para los Municipios}

Tienen como finalidad adquirir tierras vacantes para el dominio municipal, con dos objetivos: por un lado, que el Estado constituya una reserva que pueda incorporarse a proyectos de desarrollo, sea de forma unilateral o en consorcio con el privado (Larangeira, 2004), y por el otro, poder intervenir en los mercados de suelo. Entre estos se pueden mencionar los siguientes:

- Compensación de deudas: los gobiernos pueden invitar a los propietarios de tierras vacantes deudores de impuestos (municipales o provinciales) a compensar su deuda a cambio de tierras.

- Juicio de apremios: este procedimiento judicial constituye un mecanismo para que los municipios puedan demandar judicialmente el pago de los tributos adeudados por los propietarios de tierras vacantes (Lanfranchi et al., 2018).

- Prescripción administrativa [7]: permite la regularización dominial de la tierra vacante (ociosa o abandonada) sobre la que los municipios ejercían derechos posesorios (Scatolini, 2013), en particular en los casos donde no existe un propietario o un poseedor de tierra.

- Acción declarativa de vacancia: permite incorporar al dominio municipal tierras vacantes abandonadas, que no sean reclamadas por sus dueños (Paolinelli et al., 2017).

[7] Ley Nacional № 24.320 
- Traspaso de tierras: es posible traspasar el dominio de tierras vacantes entre distintos niveles de gobierno.

- Donación: es una forma de adquisición del dominio, que puede ser pública o privada, y requiere de dos cosas, la oferta de donación y la aceptación.

- Expropiación: "pueden ser objeto de expropiación todos los bienes convenientes o necesarios para la satisfacción de la 'utilidad pública', cualquiera sea su naturaleza jurídica, pertenezcan al dominio público o al dominio privado, sean cosas o no" (Ley Nacional de Expropiaciones № 21499). De este modo, este instrumento puede ser útil para la incorporación de tierra vacante al patrimonio del Estado para la satisfacción de esa utilidad pública mencionada. Teniendo en cuenta que para expropiar es necesario consolidar un pago por parte del Estado hacia el propietario de la tierra, esto se convierte en un problema en Argentina, ya que los que expropian son, en muchos casos, los Estados municipales, que no cuentan con grandes recursos económicos.

- Cesión de tierra: le permite al Estado captar tierra ya que, al crear o ampliar núcleos urbanos, los propietarios de los predios involucrados deben cederle gratuitamente las superficies destinadas a espacios circulatorios, verdes, libres y públicos (que pasan al dominio público del Estado) y a reservas para la localización de equipamiento comunitario de uso público (que pasan al dominio privado del Estado). Es un instrumento que aporta a la funcionalidad de los espacios urbanos pues, de no aplicarse, tendría que ser el Estado el generador de estos elementos de soporte necesarios en las ciudades.

- Ocupación de tierras vacantes abandonadas: a partir de ordenanzas municipales se puede establecer la obligatoriedad de mantener dichas tierras en condiciones de limpieza, libres de pastizales, basura, o cualquier elemento que signifique un peligro real o potencial para la salud y/o para el medio ambiente, y/o un daño a la comunidad. En caso de no cumplir, se puede disponer su ocupación, ya que por razones de higiene o seguridad afectan los intereses de la comunidad.

En muchos casos, los instrumentos de adquisición anteriormente mencionados se utilizan de forma independiente y no coordinada por los gobiernos locales, lo que genera la adquisición de tierras de pequeños tamaños dispersos en la ciudad, que no tienen planificación o sistematización. Para que una utilización de este tipo de instrumento sea beneficioso para la gestión, es necesario direccionar las acciones y coordinar con los organizamos de registro de propiedad inmueble.

\section{Instrumentos para administrar Tierra Vacante}

Los instrumentos para administrar son aquellos que le permiten al municipio administrar las tierras vacantes localizadas dentro de sus límites. Entre ellos se encuentran los siguientes:

- Registro oficial de tierras vacantes: es la base de datos para sistematizar la información catastral, dominial, registral, y de características (localización, dimensiones, infraestructura, entre otros aspectos) de la tierra vacante de todo el municipio, tanto de propiedad privada como pública, que debe contener información actualizada periódicamente. Este registro es la base fundamental que necesitan los gobiernos locales para poder aplicar los instrumentos.

- Banco de tierras: administra los bienes inmuebles públicos municipales, entre los que se incluyen las tierras vacantes. Conforma una unidad de servicio útil para la aplicación de otros instrumentos de gestión y posibilita la disponibilidad de tierras para la implementación de estrategias de desarrollo urbano, económico y social. A pesar de que su funcionamiento podría asimilarse a una empresa inmobiliaria, constituye una reserva física de carácter estratégico para el desarrollo urbano, con la diferencia sustancial de no tener como fin la generación de ganancias, sino el bienestar de la sociedad en su conjunto. De esta manera, el municipio cuenta con recursos propios para satisfacer las demandas de suelo y para incidir en el mercado (Uccellatore et al., 2015).

- Fondo de desarrollo urbano: es una cuenta que sirve para financiar las inversiones en bienes públicos con fines de desarrollo urbano local, que se alimenta de los recursos generados a través de la aplicación de instrumentos tales como convenios urbanísticos, impuestos por tierras sin uso, contribución por mejoras, contribución por valorización del suelo, reajuste de tierras, compensación de deudas, donaciones y multas (Uccellatore et al., 2015). Los municipios pueden combinar la administración de bienes del banco de tierras con la gestión de este fondo. 


\begin{tabular}{|c|c|c|}
\hline \multicolumn{2}{|l|}{ OBJETIVO } & INSTRUMENTO \\
\hline \multirow[t]{7}{*}{ Para movilizar } & \multirow[t]{2}{*}{ Para exigir } & Edificación y/o parcelamiento obligatorio \\
\hline & & Declaración de zonas de desarrollo prioritario \\
\hline & \multirow[t]{4}{*}{ Para facilitar } & Reajuste de tierras \\
\hline & & Consorcio urbanístico \\
\hline & & Lote con servicios \\
\hline & & Concesión de uso \\
\hline & Para incentivar & Gravámenes (tasas, impuestos, multas) \\
\hline \multirow{8}{*}{$\begin{array}{l}\text { Para adquirir para el } \\
\text { dominio municipal }\end{array}$} & & Donaciones \\
\hline & & Traspaso de tierras entre niveles del Estado u organismos \\
\hline & & Expropiación \\
\hline & & Compensación de deudas \\
\hline & & Juicio de apremios \\
\hline & & Prescripción administrativa \\
\hline & & Ocupación de tierras abandonadas \\
\hline & & Cesión \\
\hline \multirow[t]{3}{*}{ Para administrar } & & Registro oficial de tierras vacantes \\
\hline & & Banco de tierras municipal \\
\hline & & Fondo de desarrollo urbano \\
\hline \multirow[t]{5}{*}{ Para planificar } & & Participación municipal en las valorizaciones inmobiliarias \\
\hline & & Plan urbano territorial \\
\hline & & Planes particularizados \\
\hline & & Normativa de regulación \\
\hline & & Zonas especiales de interés social \\
\hline
\end{tabular}

Tabla 1. Instrumentos para la gestión de tierra vacante en ciudades argentinas. Fuente: Elaboración propia.

\section{Instrumentos para planificar la Tierra Vacante al interior de los Procesos de Crecimiento Urbano}

Por último, existen instrumentos para planificar, de acuerdo con el crecimiento urbano y las tierras vacantes; entre ellos se pueden mencionar los siguientes:
- Participación municipal en las valorizaciones inmobiliarias: al momento de pensar el desarrollo de áreas vacantes es muy importante tener en cuenta la valorización que se genera en la zona de influencia del proyecto por las distintas intervenciones que se lleven adelante. Este instrumento puede ser utilizado para distribuir la renta urbana y democratizar el acceso al suelo, teniendo como principio que el incremento del precio que puede tener una 
tierra vacante es socialmente producido (por la acción del Estado) y, por lo tanto, debe ser capturado y revertido para el beneficio de la comunidad. Este incremento puede ser capturado de diversas maneras: impuestos, gravámenes, contribución por mejoras, aportes urbanísticos (como la cesión de tierra), construcción de vivienda social, entre otros. Sin embargo, a excepción de algunos, estos métodos son muy difíciles de aplicar en Argentina debido a la cultura patrimonialista que rodea la propiedad privada.

- Plan urbano territorial: plantea las directrices generales para el crecimiento urbano en general y la tierra vacante en particular. Su elaboración es necesaria para la definición de otros instrumentos que contribuyen a la ejecución de las políticas urbanísticas. Por tratarse de una herramienta realizada en un momento determinado, y por establecer parámetros generales, es necesario que contenga mecanismos que le permitan estar sujeta a revisiones y modificaciones.

- Planes particularizados: para el desarrollo de propuestas integrales para la renovación, reestructuración y consolidación de áreas vacantes.

- Normativa de regulación: es la expresión jurídica del plan urbano y regula lo que se puede hacer sobre cada tierra vacante, es decir, dónde puede crecer, con qué indicadores urbanísticos debe hacerlo, cómo se puede subdividir y qué usos pueden darse en ella.

- Zonas especiales de interés social: facilitan el acceso al suelo a sectores populares; son delimitaciones de predios vacantes en áreas aptas para urbanizar, que pasan a ser objeto de estudio, intervención y reglamentación específicos (Reese, 2011).

\section{Reflexiones Finales}

Como se pudo observar, los instrumentos para accionar sobre la vacancia de suelo en las ciudades argentinas son variados, lo que pone de manifiesto la capacidad y potencialidad que puede tener el Estado para actuar sobre el fenómeno. De este modo, "todos los municipios del país cuentan con potestades para regular el mercado de suelo. Sin embargo, por debilidades institucionales, carencia de conocimientos técnicos o falta de instrumentos vigentes, la mayoría de ellos no las utiliza o lo hace parcialmente" (Lanfranchi et al., 2018, p. 242). Sin embargo, fue el mismo Estado el que los creó, producto de prácticas pasadas. De este modo se pone en evidencia la inacción del Estado en esta materia.

En muchos casos, a pesar del creciente impulso de los Estados municipales de muchas ciudades argentinas en la formulación de estos instrumentos para la regulación del suelo en general, y del vacante en particular, "su aplicación efectiva (...) resulta incierta, en la medida que éstas tropiezan con regímenes legales (...) que protegen los derechos de propiedad individual por encima del interés social y enfrentan la oposición que ejercen los intereses ligados al capital inmobiliario" (Cuenya, 2017).

En este sentido, la tierra vacante puede ser vista como uno de los principales problemas de las ciudades en la actualidad y al mismo tiempo como un desafío que representa innumerables oportunidades para el redireccionamiento de los actuales procesos de crecimiento urbano. Así, se hace necesario que las tierras vacantes formen parte de las prioridades de intervención del Estado, enmarcándose en políticas que las consideren. Por eso se entiende que la capacidad de gestión del Estado para dar respuestas adecuadas a la problemática de la vacancia del suelo, de acuerdo con las demandas de las ciudades argentinas, necesita articular estos instrumentos, para poder utilizarlos y combinarlos eficientemente. A su vez, es necesario diseñar marcos normativos nacionales como una forma de incrementar las capacidades para implementar una política urbana nacional, así como la coordinación institucional, en relación con la articulación, por ejemplo, entre organismos de registro, de planificación urbana y organismos fiscales.

De este modo, con el fin de redireccionar los actuales procesos de crecimiento urbano, la gestión de la tierra vacante se establece como un aspecto fundamental de la planificación. El Estado debe tener un rol activo en la orientación de ese crecimiento, diseñando políticas integrales que promuevan la utilización de tierras vacantes localizadas en áreas estratégicas, aprovechando la infraestructura existente, consolidando el suelo actualmente urbanizado, en lugar de seguir extendiendo la ciudad sin límites. Para este fin es necesario modificar los patrones que promueven la especulación, para desalentar la retención de tierras vacantes ociosas, disminuyendo los diferenciales de renta que lo incentivan. 


\section{Referencias}

ABRAMO, P. (2012). La ciudad com-fusa: mercado y producción de la estructura urbana en las grandes metrópolis latinoamericanas. Revista EURE - Revista de Estudios Urbano Regionales, 38(114), 35-69. https://www.eure.cl/index.php/ eure/article/view/68

BAER, L. (2011). El mercado de suelo formal de la Ciudad de Buenos Aires en su contexto metropolitano. Dinámica de precios de terrenos, desarrollo inmobiliario y acceso a la vivienda en la década de dos mil. [Tesis de doctorado, Universidad de Buenos Aires]. http://catalogosuba.sisbi.uba.ar/vufind/ Record/201603310123092400/Details

BANCO MUNDIAL (2018). Población urbana. Perspectivas de la urbanización mundial. https://datos. bancomundial.org/indicador/sp.urb.totl.in.zs

BARENBOIM, C. (2013). Mercado Inmobiliario, Normativa e Impacto Territorial: Rosario y su periferia. Universidad Nacional de Rosario.

BORJA, J. Y CASTELLS, M. (2000). Local y global: la gestión de las ciudades en la era de la información. Santillana Ediciones Generales.

COMISIÓN ECONÓMICA PARA AMÉRICA LATINA Y EL CARIBE - CEPAL (2017). Pese a avances recientes, América Latina sigue siendo la región más desigual del mundo. https://www.cepal. org/es/comunicados/cepal-pese-avances-recientesamerica-latina-sigue-siendo-laregion-masdesigual-mundo

CLICHEVSKY, N. (2007). La tierra vacante "revisitada". Elementos explicativos y potencialidades de utilización. Revista Cuaderno Urbano, 6(6), 195-219. http://dx.doi.org/10.30972/ crn.661023

CORTIZO, D. (2018). Tierra vacante y expansión urbana. Aporte teórico metodológico para una futura intervención desde un enfoque sostenible. El caso del Partido de La Plata. [Tesis de maestría. Universidad Nacional de La Plata]. http://sedici.unlp.edu.ar/ handle/10915/72414
CUENYA, B. (2017). Nuevos enfoques y herramientas para la regulación del suelo en Argentina. Una lectura desde la perspectiva de la reforma urbana. Revista Digital Café de las ciudades. https://cafedelasciudades.com.ar/sitio/contenidos/ ver/41/nuevos-enfoques-y-herramientas-para-laregulacion-del-suelo-en-argentina.html

DE MATTOS, C. (2002). Transformación de las ciudades latinoamericanas ¿Impactos de la globalización? Revista EURE - Revista de Estudios Urbano Regionales, 28 (85). http://dx.doi. org/10.4067/S0250-71612002008500001

ESCALES, V; GHELFI, F; PERELMAN, M.; TORDINI, X. (EDS.) (2017). Herramientas para aplicar la Ley de Acceso Justo al Hábitat. CELS [Archivo PDF]. https://www.cels.org.ar/web/ publicaciones/herramientas-para-aplicar-la-leyde-acceso-justo-al-habitat/

FREDIANI, J. (1-2 DE OCTUBRE DE 2014). La tierra vacante al interior del proceso de expansión urbana en el Gran La Plata. [Ponencia] XVI Jornadas de Investigación del Centro de Investigaciones Geográficas y del Departamento de Geografía. UNLP. http://www.memoria.fahce.unlp.edu.ar/ trab_eventos/ev.4100/ev.4100.pdf

LANFRANCHI, G.; CORDARA, C.; DUARTE, J.I.; GIMENEZ HUTTON,T.; RODRIGUEZ, S.; FERLICCA, F. (2018). ¿Cómo crecen las ciudades argentinas? Estudio de la expansión urbana de los 33 grandes aglomerados. CIPPEC [Archivo PDF] https://www.cippec.org/publicacion/como-crecenlas-ciudades-argentinas-estudio-de-la-expansionurbana-de-los-33-grandes-aglomerados/

LARANGEIRA, A. (2004). Tierra Vacante en las ciudades de América Latina: Desafíos y Oportunidades. Lincoln Institute of Land Policy.

LUCO, C.; SIMIONI, D. (2001). Dinámica de valorización del suelo en el Área Metropolitana del Gran Santiago y desafios del financiamiento urbano. CEPAL; ECLAC.
GOYTIA，C.; CRISTINI， M. (EDS.) (2017). Argentina 2030, Diagnóstico sobre ciudades y desarrollo urbano. Centro de Investigación de Políticas Urbanas y Vivienda. Universidad Torcuato Di Tella y Jefatura de Gabinete, Presidencia de la Nación. https://www.utdt.edu/ver_contenido.php?id_ contenido=19364\&id_item_menu=31030

PAOLINELLI, J.; GUEVARA, T., OGLIETTI, G.; NUSSBAUM, M.A. (2017). Lotes sin dueño. Derecho de propiedad y abandono como problema urbano. Editorial UNRN.

PERELMAN, M; GHELFI, F; ESCALES, V. (EDS.) (2017). Consenso Nacional para un Hábitat Digno Diez propuestas de políticas públicas. CELS. [Archivo PDF]. https://www.cels.org.ar/web/publicaciones/ consenso-nacional-para-un-habitat-digno-diezpropuestas-de-politicas-publicas/

REESE, E. (9 DE NOVIEMBRE DE 2018). La Ley de Acceso Justo al Hábitat [Discurso principal]. Charlas Acceso Justo al Hábitat. CAPBAUNO, La Plata, Argentina.

REESE, E. (2011). Instrumentos de gestión urbana, fortalecimiento del rol del municipio y desarrollo con equidad. Revista digital Café de las ciudades. https://cafedelasciudades.com.ar/carajillo/10_art4. htm

SCATOLINI, L. (2013). Prescripción administrativa: Instrumento de gestión de suelo. [Archivo PDF] Documento de trabajo del Lincoln Institute of Land Policy. https://www.lincolninst.edu/sites/default/ files/pubfiles/scatolini-wp14ls2sp-full_0.pdf

UCCELlATORE, M.; DELBÓN, G. KRIVOCAPICH，G.; HAGEDORN，T. (2015) Financiamiento de Infraestructura Urbana. Criterios para un modelo de gestión público-privado. Secretaría de Asuntos Municipales, Ministerio del Interior y Transporte. Argentina. https://www.mininterior gov.ar/municipios/pdfs/SAM_07_v.2015 INFRAESTRUCTURA_URBANA_CMYK.pdf 